\title{
Extra-spinal incidental findings at lumbar spine MRI in the general population: a large cohort study
}

\author{
Carlo Cosimo Quattrocchi • Alessandro Giona • \\ Alberto Corrado Di Martino • Yuri Errante • \\ Laura Scarciolla • Carlo Augusto Mallio • \\ Vincenzo Denaro • Bruno Beomonte Zobel
}

Received: 11 January 2013 /Revised: 9 February 2013 / Accepted: 11 February 2013 / Published online: 2 March 2013

(C) The Author(s) 2013. This article is published with open access at Springerlink.com

\begin{abstract}
Objective To determine the prevalence of clinically and non-clinically relevant extra-spinal incidental findings (IF) in patients undergoing magnetic resonance imaging (MRI) of the lumbar spine and to evaluate the rate of undetected findings in archived radiological reports.

Methods A retrospective search of patients undergoing lumbar spine MRI from January 2006 to December 2010 was conducted. By means of randomisation, we retrospectively reviewed 3,000 lumbar spine MRI examinations. Extraspinal abnormalities were classified according to a modified CT Colonography Reporting and Data System (C-RADS). We retrospectively compared our structured approach with the archived MRI reports as it regarded the detection of extra-spinal IF to estimate non-detection rates.

Results By means of the structured approach used, extraspinal findings were detected in 2,060 (68.6\%) of the 3,000 lumbar spine MRI examinations; 362 (17.6\%) patients had indeterminate or clinically important findings (E3 and E4) requiring clinical correlation or further evaluation. After review of the original archived radiological reports, potentially important C-RADS E3 and E4 extra-spinal IF were
\end{abstract}

C. C. Quattrocchi $(\triangle) \cdot$ A. Giona $\cdot$ Y. Errante $\cdot$ L. Scarciolla $\cdot$

C. A. Mallio - B. B. Zobel

Diagnostic Imaging, Università Campus

Bio-Medico di Roma, via Alvaro del Portillo, 21,

00128 Rome, Italy

e-mail: c.quattrocchi@unicampus.it

A. C. Di Martino $\cdot$ V. Denaro

Orthopaedics and Trauma Surgery, Centre for

Integrated Research (CIR), Università Campus

Bio-Medico di Roma, via Alvaro del Portillo, 21,

00128 Rome, Italy

C. C. Quattrocchi · A. Giona

Radiology, Fondazione San Raffaele, Cassino, FR, Italy respectively reported in 47 of the $265(17.7 \%)$ and in 8 of $74(10.8 \%)$ patients.

Conclusions Our study shows that incidental extra-spinal findings at conventional lumbar spine MRI are common but underestimated in radiological reports.

Keywords Lumbar spine $\cdot$ IF · Extra-spinal findings · Radiological report $\cdot$ Magnetic resonance imaging

\section{Abbreviations}

C-RADS CT Colonography Reporting and Data System IF Incidental findings

\section{Introduction}

Incidental findings (IF) at imaging are defined as previously undetected abnormalities that are unexpectedly discovered and are unrelated to the purpose of the examination. Lumbar spine MRI may reveal either clinically insignificant spine incidental abnormalities and/or extra-spinal IF that, at times, may even explain the patient's symptoms. The detection of such findings poses various practical and ethical issues concerning clinical management. At lumbar spine magnetic resonance imaging (MRI), despite the signal saturation bands that are used in standard international protocols to reduce the number and severity of artefacts [1], incidental abnormalities may arise from a wide range of abdominal and pelvic organs and the diseases encountered may be extremely diverse.

Picture archiving and communication systems (PACS) are aimed to improve reporting efficiency, and their introduction has resulted in an increase of the number of both reported IF and follow-up examinations [2, 3]. 
Recently, the prevalence of clinically relevant extraspinal abnormalities in 400 consecutive adult outpatients undergoing computed tomography (CT) of the lumbar spine has been reported; in this study, the authors reviewed extra-spinal pathological findings in addition to many benign IF [4].

In an earlier retrospective study, Frager et al. [5] demonstrated extra-spinal pathology at lumbar CT examinations in $22(1.45 \%)$ out of 1,517 patients. IF included retroperitoneal tumours and lymphadenopathies as well as vascular, urinary tract and gynaecological abnormalities.

Another study focused on spinal abnormalities reported on IF of patients undergoing MRI because of suspected herniated intervertebral disk disease of the lumbar spine [6].

Although review articles or clinical case reports describing extra-spinal abnormalities at MRI of the lumbar spine have been published [7-9], we are not aware of any large cohort study exploring the prevalence and clinical importance of extra-spinal findings at conventional lumbar spine MRI. In this study, by means of a structured reporting approach, we systematically searched for the presence of clinically and non-clinically relevant extraspinal IF in patients undergoing MRI of the lumbar spine, and estimated the rate of undetected findings in archived radiological reports.

\section{Materials and methods}

\section{Study design}

A retrospective search of the entire digital archive of patients undergoing lumbar spine MRI was conducted, after obtaining informed consent, from January 2006 to December 2010 in our institution. The study was reviewed and approved by the institution's ethical committee. Patients with known history of malignancy with or without spine involvement and patients with repeated lumbar spine MR examinations over the 2006-2010 period were excluded. Both conditions would have generated a bias on estimates of the prevalence of the extra-spinal MRI findings. Out of the entire archive, 10,254 lumbar spine MR examinations were considered eligible for the study. By means of random sampling, obtained by using the Research Randomizer tool (http://www.randomizer.org), we included 3,000 lumbar spine MRI examinations in the study.

\section{MRI}

MRI was performed on a 1.5-T MR imaging unit (Avanto, Siemens, Erlangen, Germany), with the 24-element design Spine Matrix coil integrated into the patient table. The MRI protocol was conducted as follows: sagittal turbo spin echo
T2-weighted sequences (slice thickness, $4.0 \mathrm{~mm}$; echo train length, 7; intersection gap, $1.0 \mathrm{~mm}$; matrix size, $512 \times 192$; field of view, $32 \times 32 \mathrm{~cm}$; TR/TE, 4,720-5,500/90-100 ms; read out bandwidth, $191 \mathrm{~Hz} / \mathrm{Px}$ ); sagittal T1-weighted sequences (slice thickness, $4.0 \mathrm{~mm}$; echo train length, 7; intersection gap, $1.0 \mathrm{~mm}$; matrix size, $512 \times 192$; field of view, $32 \times 32 \mathrm{~cm}$; TR/TE, $400 / 8 \mathrm{~ms}$; read out bandwidth, $191 \mathrm{~Hz} / \mathrm{Px}$ ); axial turbo spin echo T2-weighted sequences (slice thickness, $3.0 \mathrm{~mm}$; echo train length, 19; intersection gap, $1.0 \mathrm{~mm}$; matrix size, $448 \times 225$; field of view, $25 \times$ $19.5 \mathrm{~cm}$; TR/TE, 4,600-5,150/100-110 ms; read-out bandwidth, $120 \mathrm{~Hz} / \mathrm{Px}$ ).

\section{Data analysis}

MR images were reviewed and interpreted by two radiologists, one with 9 years of experience (C.C.Q., neuroradiologist) and the other with 5 years of experience (A.G., musculoskeletal radiologist) in reading spine MR images. Extra-spinal findings were reported by consensus in a structured database built on Microsoft Excel, using acronyms to classify findings according to specific organs and/or systems (e.g. vascular system, lymphatic system, kidney, uterus, ovaries, etc.) (Table 1). The legend at the bottom of Table 1 defines some findings such as criteria to differentiate aortic dilation from aneurysm or to consider a finding as benign.

Because of the variability of the classification systems in the literature, we decided also to include a classification of the extra-spinal abnormalities based on the CT Colonography Reporting and Data System (C-RADS), as previously reported $[4,10]$. Only anatomic variants were recorded for the C-RADS E1 category (normal examination results or anatomic variants); the C-RADS E2 category was assigned to clinically unimportant findings for which no further work-up or assessment was indicated (e.g. renal cyst, diverticulosis); the C-RADS E3 findings were indeterminate, incompletely characterised, but likely benign findings for which clinical correlation and further work-up could be performed if indicated (e.g. minimally complex renal cyst); the C-RADS E4 category designated potentially important findings requiring further work-up and communication to the referring physician, as per accepted practice guidelines (e.g. solid renal mass, abdominal aortic aneurysm). If a patient had multiple extraspinal findings, the MRI study was classified according to the most clinically important abnormality.

Finally, we retrospectively compared our structured approach with the archived MRI reports as regards the detection of extra-spinal IF to estimate non-detection rates. Patient demographic data, MR findings and adapted CRADS classifiers were loaded and archived in the database and descriptive statistics were performed. 
Table 1 List of IF classified according to the organ/system involved

\begin{tabular}{|c|c|}
\hline Organ/system & Finding \\
\hline Vascular System & $\begin{array}{l}\mathrm{AP}=\text { atheromasic plaque } \\
\mathrm{AD}=\text { aortic Dilation }^{\mathrm{a}} \\
\mathrm{AA}=\text { aortic Aneurysm }^{\mathrm{b}} \\
\mathrm{V}=\text { pelvic Varicocele }\end{array}$ \\
\hline Lymphatic System & $\mathrm{L}=$ enlarged Lymph nodes ${ }^{\mathrm{c}}$ \\
\hline Kidney & 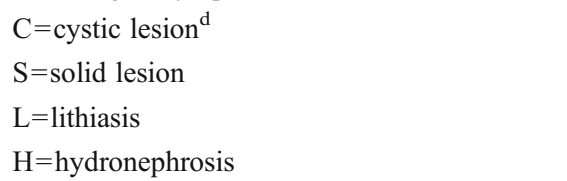 \\
\hline Uterus & $\begin{array}{l}\mathrm{CB}=\text { cystic benign lesion }^{\mathrm{d}} \\
\mathrm{SB}=\text { solid benign lesion }^{\mathrm{e}} \\
\mathrm{A}=\text { anatomic abnormality }^{\mathrm{f}} \\
\mathrm{UD}=\text { cavity dilation }^{\text {can }}\end{array}$ \\
\hline Ovaries & $\begin{array}{l}\mathrm{CL}=\text { cystic Lesion }^{\mathrm{d}} \\
\mathrm{SL}=\text { solid lesion } \\
\mathrm{PS}=\text { post-surgery }\end{array}$ \\
\hline $\begin{array}{l}\text { Prostate } \\
\text { Bladder }\end{array}$ & $\mathrm{PL}=$ bladder wall thickening or prostatic lesion \\
\hline Bowel & $\begin{array}{l}\mathrm{D}=\text { diverticulosis } \\
\mathrm{CT}=\text { colo-rectal wall thickening }\end{array}$ \\
\hline Liver & $\begin{array}{l}\mathrm{SC}=\text { Simple } \text { cysts }^{\mathrm{d}} \\
\mathrm{HL}=\mathrm{T} 2 \text { hyperintense lesion } \\
\mathrm{BL}=\text { biliary lithiasis } \\
\mathrm{BD}=\text { biliary duct dilation }\end{array}$ \\
\hline Spleen & $\mathrm{AS}=$ accessory spleen \\
\hline Adrenal glands & $\begin{array}{l}\mathrm{AH}=\text { hyperplasia } \\
\mathrm{ASL}=\text { solid lesion }\end{array}$ \\
\hline Fluid & $\mathrm{F}=$ abdominal-pelvic fluid \\
\hline
\end{tabular}

${ }^{\text {a }}$ Transverse diameter from 2.6 to $3.0 \mathrm{~cm}$

${ }^{\mathrm{b}}$ Transverse diameter more than $3.0 \mathrm{~cm}$

${ }^{\mathrm{c}}$ Lymph nodes (pre-sacral in all cases) were considered enlarged with the largest diameter greater than $5 \mathrm{~mm}$

${ }^{\mathrm{d}}$ Kidney, liver, uterus and ovary lesions that showed net hyperintensity on T2-weighted and net hypointensity on T1-weighted images, with well-defined margins were considered presumably benign without the need of further work-up

${ }^{\mathrm{e}}$ Solid benign lesions were classifid as E2 if homogeneously hypointense on T1- and T2-weighted images, suggesting fibromiomas

${ }^{\mathrm{f}}$ Anatomical abnormalities were: retroverse uterus, uterus didelphis, bicornuate uterus, septated uterus

${ }^{\mathrm{g}}$ Colorectal wall thickening was not defined because colorectal enema was not performed; any wall thickening considered suspicious by consensus of the two observers was included in the study

\section{Results}

The mean age of our study population was 59.3 years (range, 16-91 years), comprising 1,453 (48.4\%) male and $1,547(51.6 \%)$ female subjects.
Extra-spinal findings were noted in 2,060 (68.7\%) of the 3,000 patients, comprising 595/3,000 (19.8\%) men and 1,465/3,000 (48.8\%) women.

Anatomic variants (e.g. retroverse uterus, duplicated collecting system), classified as C-RADS E1 category, were detected in 49/3,000 (1.6\%) patients (2 males, 47 females). Out of this group, 24 patients also had a C-RADS E2 finding that required no further work-up.

Table 2 summarises the results of the C-RADS E2, E3, and E4 extra-spinal findings. The largest portion of extraspinal findings was classified in the C-RADS E2 category $(57.4 \%, 1,721 / 3,000)$, with simple renal cysts $(42.5 \%$, $732 / 1,721)$, colon diverticulosis $(20.4 \%, 351 / 1,721)$, ovarian simple cysts $(12.8 \%, 221 / 1,721)$ and uterine solid $(12.7 \%, 219 / 1,721)$ or cystic $(6.8 \%, 117 / 1,721)$ benign lesions being the most prevalent findings.

Among all patients, 339 patients (11.3\%, 339/3,000) were included in the C-RADS E3 and E4 groups. The most prevalent finding in the E3 group was the presence of abdominal-pelvic fluid $(77.0 \%, 204 / 265)$. Out of these, $169(82.8 \%, 169 / 204)$ were women presenting fluid collection in the Douglas pouch. Seventy-four $(2.5 \%)$ of the 3,000 patients were categorised as C-RADS E4 (Table 2). The most common findings were enlarged lymph nodes $(38 / 74,51.3 \%)$, prostatic lesion or bladder wall thickening $(15 / 74,20.3 \%)$ and abdominal aortic aneurysms $(11 / 74$, $14.9 \%)$. In seven cases $(7 / 74,9.4 \%)$ colorectal wall thickening was observed (Fig. 1 and 2).

After revision of the archived radiological reports, extraspinal findings were detected in 217/3,000 patients (7.2\%). According to the C-RADS classification, potentially important C-RADS E3 and E4 extra-spinal IF were respectively reported only in 47/265 (17.7\%) and in 8/74 (10.8\%) patients (Table 3). Simple renal cysts were the least reported extra-spinal IF (30/732 or $4.0 \%$ of cases), while among the most frequent findings, uterine solid benign lesions were originally reported in a relatively high number of cases (52/219, $23.7 \%)$.

In the C-RADS E4 group, colo-rectal wall thickening suspicious for cancer was originally reported in $42.8 \%$ of the cases (3/7). Aortic aneurysms often remained undetected $(1 / 11,9.0 \%)$.

When clinically relevant extra-spinal E4 IF were identified and reported at the time of the MRI scan, radiologists recommended further clinical and/or radiological investigation in all cases $(8 / 8)$.

In 22 patients we found bladder or colic wall thickening suspicious for cancer; endoscopic examinations were conducted in our hospital on nine of these cases, confirming the diagnosis of malignancy in seven patients (four bladder cell transitional carcinoma, two colorectal cancers and one prostatic cancer extended to the bladder). 
Table 2 Summary of IF, classified according to the modified C-RADS classification

\begin{tabular}{|c|c|c|c|c|c|}
\hline Organ/system & Finding & Number & Rate $(\%)$ & Men & Women \\
\hline \multicolumn{6}{|c|}{ C-RADS E2: clinically unimportant findings - no further work-up indicated } \\
\hline \multirow[t]{2}{*}{ Vascular system } & Atheromasic plaque & 11 & 0.5 & 4 & 7 \\
\hline & Pelvic varicocele & 21 & 1.0 & 0 & 21 \\
\hline \multirow[t]{2}{*}{ Kidney } & Cystic Lesion & 732 & 35.5 & 321 & 411 \\
\hline & Lithiasis & 2 & 0.1 & 1 & 1 \\
\hline \multirow[t]{3}{*}{ Uterus } & Solid benign lesion & 219 & 10.6 & 0 & 219 \\
\hline & Cystic benign lesion & 117 & 5.7 & 0 & 117 \\
\hline & Anatomic abnormality & 12 & 0.6 & 0 & 12 \\
\hline \multirow[t]{2}{*}{ Ovaries } & Cystic Lesion & 221 & 10.7 & 0 & 221 \\
\hline & Post-surgery & 1 & 0.1 & 0 & 1 \\
\hline Prostate & Post-surgery & 15 & 0.7 & 15 & 0 \\
\hline \multicolumn{6}{|l|}{ Bladder } \\
\hline Bowel & Diverticulosis & 351 & 20.4 & 142 & 209 \\
\hline \multirow[t]{3}{*}{ Liver } & Simple cysts ${ }^{\mathrm{a}}$ & 6 & 0.3 & 2 & 4 \\
\hline & Biliary Lithiasis & 6 & 0.3 & 1 & 5 \\
\hline & Biliary duct dilation $(<6 \mathrm{~mm})$ & 5 & 0.3 & 3 & 2 \\
\hline Spleen & Accessory spleen & 2 & 0.1 & 1 & 1 \\
\hline Total E2 & & 1,721 & 83.5 & 490 & 1,231 \\
\hline \multicolumn{6}{|c|}{ C-RADS E3: likely unimportant findings, incompletely characterised } \\
\hline Vascular system & Aortic dilation & 11 & 0,6 & 8 & 3 \\
\hline \multirow[t]{2}{*}{ Kidney } & Hydronephrosis & 4 & 0.2 & 1 & 3 \\
\hline & Solid lesion & 4 & 0.2 & 1 & 3 \\
\hline Uterus & Uterine cavity dilation & 15 & 0.7 & 0 & 15 \\
\hline Liver & $\mathrm{T} 2$ hyperintense lesion & 27 & 1.3 & 14 & 13 \\
\hline Fluid & Abdominal-Pelvic Fluid & 204 & 9.9 & 35 & 169 \\
\hline Total E3 & & 265 & 12.9 & 59 & 206 \\
\hline \multicolumn{6}{|c|}{ C-RADS E4: potentially important findings } \\
\hline Vascular system & Aortic aneurysms & 11 & 0.6 & 6 & 5 \\
\hline Lymphatic system & Enlarged lymph nodes & 38 & 1.8 & 26 & 12 \\
\hline Uterus & Uterine solid lesion & 2 & 0.1 & 0 & 2 \\
\hline Prostate & Bladder wall thickening or prostatic lesion & 15 & 0.7 & 11 & 4 \\
\hline \multicolumn{6}{|l|}{ Bladder } \\
\hline Bowel & Colo-rectal wall thickening & 7 & 0.3 & 3 & 4 \\
\hline Adrenal glands & Solid lesion & 1 & 0.1 & 0 & 1 \\
\hline Total E4 & & 74 & 3.6 & 46 & 28 \\
\hline Total & & 2,060 & 100.0 & 595 & 1,465 \\
\hline
\end{tabular}

Although we identified two suspicious uterine lesions, further examination was not performed in our hospital and we miss information on outcome in those patients.

\section{Discussion}

Our study shows that incidental asymptomatic extra-spinal findings at conventional lumbar spine MRI are common in the general population but are under-represented in the radiological reports of our cohort.
The prevalence of incidental extra-spinal findings in our study $(68.6 \%)$ was higher than the $40 \%$ prevalence reported on lumbar spine CT examinations [4], and higher than the extra-colonic abdominal findings documented in previous studies on CT colonography $[11,12]$. We believe that the higher contrast resolution of MRI images on the pelvis may explain the difference with previous studies conducted on CT scans. The higher reported prevalence of pelvic benign lesions, such as uterine leiomyomas and ovarian cysts, and the high prevalence of abdominal-pelvic fluid collections in women support this idea. Nephrolithiasis was rarely detected 

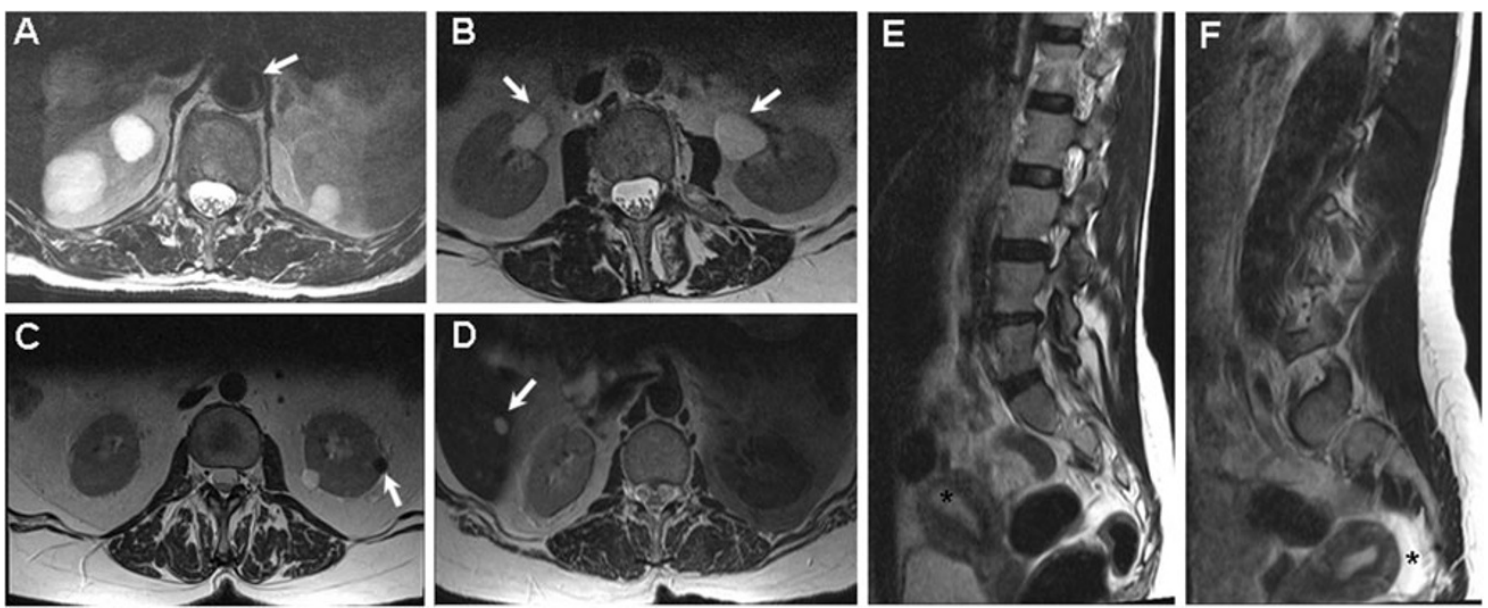

Fig. 1 Example images of C-RADS E3 (likely unimportant, incompletely characterised) IF. a Aortic dilation on axial T2 weighted images (white arrow); b bilateral hydronephrosis on axial $\mathrm{T} 2$ weighted images (white arrows); c solid T2 hypointense cortical lesion of the left kidney (white arrow); d T2 hyperintense focal hepatic lesion (white arrow); uterine cavity dilation (white asterisk); f pelvic fluid in the Douglas pouch (white asterisk)

( $0.1 \%$ of the population) compared with the expected $13.9 \%$ prevalence of previous $\mathrm{CT}$ studies [13]; this may be explained by a lower sensitivity of MR compared with $\mathrm{CT}$ and by the incomplete coverage of the kidneys in the field of view of lumbar spine MRI.

The prevalence of clinically relevant vascular extraspinal findings in our study was inferior to that described by Gouliamos et al. [14], who found abdominal aortic aneurysms in $3 \%$ of patients undergoing lumbar spine CT for low-back pain and to that of Lee et al. [4]. These differences may be mainly due to the use of signal saturation bands positioned anterior to the spine, thus limiting the complete evaluation of the abdominal aorta.

The clinical impact of IF on patient health outcome is not certain, and discussion is open [15], but it is worth remembering that an incidental finding may, at times, be more significant than the suspected disease that prompted imaging. As we reported, the majority of findings in this study were classified as C-RADS E2 and, as such, were clinically not relevant. However, the structured approach led us to detect C-RADS E3 and E4 findings in $16.5 \%$ of the patients.

Among the C-RADS E3 findings, T2 hyperintense hepatic lesions are often related to benign pathologies, such as simple hepatic cysts or hepatic haemangiomas; however, further examination is required to distinguish between the benign common abnormalities and pathological liver lesions that could impact with health patient outcome.

As regards hydronephrosis, although the aetiology may remain undefined on lumbar MRI images, its presence may guide the clinician to identify and treat the underlying cause prior to permanent loss of the kidney function.
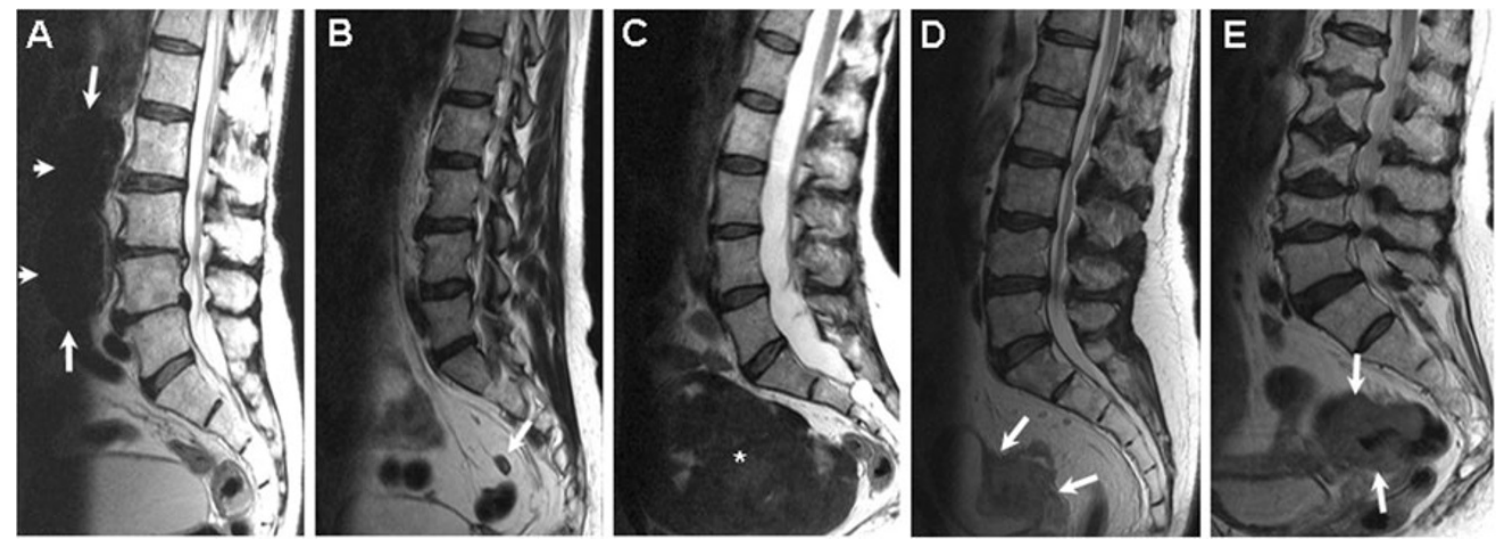

Fig. 2 Example images of C-RADS E4 (potentially important) IF. a Aortic aneurysm with low signal due to the positioning of the saturation band on sagittal T2 weighted images (white arrows); b pre-sacral enlarged lymphnode (white arrow); c complex pelvic mass interpreted as uterine solid lesion (white asterisk); $\mathbf{d}$ a solid pelvic mass that turned out to be a T4 stage prostatic carcinoma (white arrow); e solid bowel irregular wall thickening that turned out to be a T2 stage colon carcinoma (white arrows) 
Table 3 Detection of lumbar spine MRI IF with a standard and a structured reporting approach

\begin{tabular}{|c|c|c|c|c|c|}
\hline & & $\begin{array}{l}\text { Radiological } \\
\text { Report }\end{array}$ & Structured report & Undetected findings & Non-detection \\
\hline Organ/system & Finding & Patients $(n)$ & Patients $(n)$ & Patients $(n)$ & rate \\
\hline \multicolumn{6}{|c|}{ C-RADS E2: clinically unimportant findings - no further work-up indicated } \\
\hline \multirow[t]{2}{*}{ Vascular system } & Atheromasic plaque & 0 & 11 & 11 & 1 \\
\hline & Pelvic varicocele & 6 & 21 & 15 & 0.71 \\
\hline \multirow[t]{2}{*}{ A } & Cystic Lesion & 30 & 732 & 702 & 0.96 \\
\hline & Lithiasis & 1 & 2 & 1 & 0.50 \\
\hline \multirow[t]{3}{*}{ Uterus } & Solid benign lesion & 52 & 219 & 167 & 0.76 \\
\hline & Cystic benign lesion & 22 & 117 & 95 & 0.81 \\
\hline & Anatomic abnormality & 3 & 12 & 9 & 0.75 \\
\hline \multirow[t]{2}{*}{ Ovaries } & Cystic Lesion & 23 & 221 & 198 & 0.90 \\
\hline & Post-surgery & 0 & 1 & 1 & 1 \\
\hline Prostate & Post-surgery & 3 & 15 & 12 & 0.80 \\
\hline \multicolumn{6}{|l|}{ Bladder } \\
\hline Bowel & Diverticulosis & 21 & 351 & 330 & 0.94 \\
\hline \multirow[t]{3}{*}{ Liver } & Simple cysts & 0 & 6 & 6 & 1.00 \\
\hline & Biliary Lithiasis & 1 & 6 & 5 & 0.83 \\
\hline & Biliary duct dilation $(<6 \mathrm{~mm})$ & 0 & 5 & 5 & 1 \\
\hline Spleen & Accessory spleen & 0 & 2 & 2 & 1 \\
\hline Total E2 & & 162 & 1,721 & 1,559 & 0.91 \\
\hline \multicolumn{6}{|c|}{ C-RADS E3: likely unimportant findings, incompletely characterised } \\
\hline Vascular system & Aortic dilation & 1 & 11 & 10 & 0.91 \\
\hline \multirow[t]{2}{*}{ Kidney } & Hydronephrosis & 1 & 4 & 3 & 0.75 \\
\hline & Solid lesion & 2 & 4 & 2 & 0.50 \\
\hline Uterus & Uterine cavity dilation & 4 & 15 & 11 & 0.73 \\
\hline Liver & $\mathrm{T} 2$ hyperintense lesion & 3 & 27 & 24 & 0.89 \\
\hline Fluid & Abdominal-Pelvic Fluid & 36 & 204 & 168 & 0.82 \\
\hline Total E3 & & 47 & 265 & 218 & 0.82 \\
\hline \multicolumn{6}{|c|}{ C-RADS E4: potentially important findings } \\
\hline Vascular system & Aortic aneurysms & 1 & 11 & 10 & 0.91 \\
\hline Lymphatic system & Enlarged lymph nodes & 2 & 38 & 36 & 0.95 \\
\hline Uterus & Uterine solid lesion & 0 & 2 & 2 & 1 \\
\hline Prostate & Bladder wall thickening or prostatic lesion & 2 & 15 & 13 & 0.87 \\
\hline \multicolumn{6}{|l|}{ Bladder } \\
\hline Bowel & Colo-rectal wall thickening & 3 & 7 & 4 & 0.57 \\
\hline Adrenal glands & Solid lesion & 0 & 1 & 1 & 1 \\
\hline Total E4 & & 8 & 74 & 66 & 0.89 \\
\hline Total & & 217 & 2,060 & 1,843 & 0.90 \\
\hline
\end{tabular}

As regards the C-RADS E4 category, aortic aneurysms have potentially serious clinical implications, mainly due to the risk of rupture. Enlarged lymph nodes also, both in the case of secondary involvement or primary lympho-proliferative disorders, may alter staging and/or treatment options in patients with cancer.

Pelvic tumours, either genito-urinary or gastrointestinal, were observed in our population and their staging as well as early diagnosis is crucial for patient outcome. Their incidental detection may anticipate the diagnosis before the clinical onset and potentially warrant higher survival rates, as suggested by Konnak et al. [16].

Despite it being speculative, we believe that a radiologically structured approach may improve the detection of extraspinal IF in comparison to a non-structured one. Wagner et al. [4] retrospectively analysed 2,500 radiological lumbar spine MRI reports and found 183 patients with extra-spinal IF $(7.3 \%)$. The review of 3,000 radiological MRI reports in our 
population showed $217(7.2 \%)$ patients with IF. With the structured approach, a much higher number of extra-spinal abnormalities (68.6\%) was detected, thus demonstrating the deficiency of reporting using a non-structured approach. Although we observed a slight gain in reporting rates as the clinical relevance of IF increases, an $85 \%$ non-detection rate was still found for C-RADS E3 and E4 findings.

The reasons for missing IF at lumbar spine MRI may be various. However, among others, we think that both musculoskeletal and neurological radiologists tend to focus their attention mainly on spinal pathology when detection of extra-spinal findings needs recall of their general radiology training. Moreover, extra-spinal IF are often asymptomatic and unexpected, with the complete absence of clinical data before scanning.

Thus, a common plan for the diagnosis of extra-spinal IF is mandatory and this is particularly important in this case, due to the high number of lumbar spine MRI scans performed every day worldwide. In a structured approach, radiologists should first focus attention on spinal pathology and subsequently on extra-spinal organs and systems, with special attention aimed at reporting IF, whether either unimportant, incompletely characterised (E3) or potentially of clinical importance (E4). Moreover, a particular effort should be made to suggest further examinations or clinical evaluation, especially at this time of poor communication between the radiologist and the patient. In cases of C-RADS E4 findings, a multidisciplinary approach should be elaborated with clinicians to guide the patient toward the best diagnostic/therapeutic plan.

On the other side, IF classified as C-RADS E1 and E2 are not clinically relevant and may lead to a serious waste of time by the radiologist and to unnecessary anxiety and confusion in patients. Thus, in those cases the radiologist should specify in the report that those findings are not clinically significant and do not require further attention.

The main limitation of this study was its retrospective design. We missed follow-up examinations and further confirmation of the clinical importance of most of the E3 and E4 findings. However, as the C-RADS classification system has been valuable in facilitating communication of extracolonic abnormalities [11], it may even be useful for reporting of extra-spinal findings on lumbar spine MRI. Also, to the best of our knowledge, this is the first large cohort study that evaluates the prevalence and clinical importance of extra-spinal findings at lumbar spine MRI.

According to epidemiological data in Italy, diagnostic errors are at the basis of $60 \%$ of claims against radiologists $[17,18]$. A correct reading of MRI scans without missing clinically important IF may prevent not only potentially severe consequences for the patient but also medico-legal implications for the radiologist.

Clinical judgement needs to be exercised in reporting of IF on lumbar spine MRI and guidelines are required to recommend further investigations, especially for subspecialist radiologists not confident with abdominal and pelvic imaging. The introduction of a structured reporting approach to lumbar spine MRI may increase awareness and detection rate of clinically relevant extra-spinal findings. Reporting policies have been addressed from the European Society of Radiology [19] with specific statements on reporting of IF; in the current clinical setting, in fact, the introduction of structured reports and formatted templates could have a profound impact on increasing the detection rate of IF. Guidelines for the advice of further actions to be suggested in the presence of potentially clinically relevant IF may be more difficult to set up; the adoption of multidisciplinary clinico-radiological approaches could enhance the clinical efficacy with a patient-centred and personalised management [20].

Open Access This article is distributed under the terms of the Creative Commons Attribution License which permits any use, distribution, and reproduction in any medium, provided the original author(s) and the source are credited.

\section{References}

1. Sze G (1991) Recent advances in spinal magnetic resonance imaging. Can Assoc Radiol J 42:190-198

2. Wagner SC, Morrison WB, Carrino JA et al (2002) Picture archiving and communication system: effect on reporting of IF. Radiology 225:500-505

3. Green L (2004) PACS: effect on IF. Radiol Manage 26(1):26-29

4. Lee SY, Landis MS, Ross IG et al (2012) Extraspinal findings at lumbar spine CT examinations: prevalence and clinical importance. Radiology 263:502-509

5. Frager DH, Elkin CM, Kansler F et al (1986) Extraspinal abnormalities identified on lumbar spine CT. Neuroradiology 28(1):58-60

6. Park HJ, Jeon YH, Rho MH et al (2011) IF of the lumbar spine at MRI during herniated intervertebral disk disease evaluation. AJR Am J Roentgenol 196:1151-1155

7. Kamath S, Jain N, Goyal N et al (2009) IF on MRI of the spine. Clin Radiol 64(4):353-361

8. Gebara NV, Meltzer DE (2009) Extraspinal findings on lumbar spine MR imaging. J Radiol Case Rep 3(8):5-13

9. Cademartiri F, Salamousas BV, Luccichenti G et al (2004) Posttraumatic descending aorta intramural haematoma fortuitously witnessed during a magnetic resonance examination of the spine. Acta Biomed 75:185-187

10. Zalis ME, Barish MA, Choi JR et al (2005) CT colonography reporting and data system: a consensus proposal. Radiology 236(1):3-9

11. Hara AK, Johnson CD, MacCarty RL et al (2000) Incidental extracolonic findings at CT colonography. Radiology 215(2):353-357

12. Xiong T, Richardson M, Woodroffe R et al (2005) Incidental lesions found on CT colonography: their nature and frequency. Br J Radiol 78(925):22-29

13. Durbin JM, Stroup SP, Altamar HO et al (2012) Genitourinary abnormalities in an asymptomatic screening population: findings on virtual colonoscopy. Clin Nephrol 77(3):204-210

14. Gouliamos AD, Tsiganis T, Dimakakos P et al (2004) Screening for abdominal aortic aneurysms during routine lumbar CT scan: modification of the standard technique. Clin Imaging 28(5):353-355

15. European Society of Radiology (ESR) (2012) ESR guidelines for the communication of urgent and unexpected findings. Insights Imaging 3(1):1-3 
16. Konnak JW, Grossman HB (1985) Renal cell carcinoma as an incidental finding. J Urol 134(6):1094-1096

17. Fileni A, Magnavita N (2006) A 12-year follow-up study of malpractice claims against radiologists in Italy. Radiol Med 111:1009-1022

18. Magnavita S, Magnavita G, Fileni A et al (2009) Ethical problems in radiology: medical error and disclosure. Radiol Med 114:13451355
19. European Society of Radiology (2011) Good practice for radiological reporting. Guidelines from the European Society of Radiology (ESR). Insights Imaging 2(2):93-96

20. European Society of Radiology (2011) Medical imaging in personalised medicine: a white paper of the research committee of the European Society of Radiology (ESR). Insights Imaging 2(6):621-630 